\title{
Anomalies and symmetric mass generation for Kähler-Dirac fermions
}

\author{
Nouman Butt, ${ }^{1}$ Simon Catterall $\odot,{ }^{2}$ Arnab Pradhan, ${ }^{2}$ and Goksu Can Toga $\odot^{2}$ \\ ${ }^{1}$ Department of Physics, University of Illinois at Urbana-Champaign, \\ 1110 West Green Street, Urbana, Illinois 61801, USA \\ ${ }^{2}$ Department of Physics, Syracuse University, Syracuse, New York 13244, USA
}

(Received 29 September 2021; accepted 14 October 2021; published 9 November 2021; corrected 3 August 2022)

\begin{abstract}
We show that massless Kähler-Dirac fermions exhibit a mixed gravitational anomaly involving an exact $U(1)$ symmetry which is unique to Kähler-Dirac fields. Under this $U(1)$ symmetry the partition function transforms by a phase depending only on the Euler character of the background space. Compactifying flat space to a sphere we learn that the anomaly vanishes in odd dimensions but breaks the symmetry down to $Z_{4}$ in even dimensions. This $Z_{4}$ is sufficient to prohibit bilinear terms from arising in the fermionic effective action. Four fermion terms are allowed but require multiples of two flavors of Kähler-Dirac field. In four dimensional flat space each Kähler-Dirac field can be decomposed into four Dirac spinors and hence these anomaly constraints ensure that eight Dirac fermions or, for real representations, sixteen Majorana fermions are needed for a consistent interacting theory. These constraints on fermion number agree with known results for topological insulators and recent work on discrete anomalies rooted in the Dai-Freed theorem. Our work suggests that Kähler-Dirac fermions may offer an independent path to understanding these constraints. Finally we point out that this anomaly survives intact under discretization and hence is relevant in understanding recent numerical results on lattice models possessing massive symmetric phases.
\end{abstract}

DOI: 10.1103/PhysRevD.104.094504

\section{INTRODUCTION}

The Kähler-Dirac equation gives an alternative to the Dirac equation for describing fermions in which the physical degrees of freedom are carried by antisymmetric tensors rather than spinors. These tensors transform under a twisted rotation group that corresponds to the diagonal subgroup of the usual (Euclidean) Lorentz group and a corresponding flavor symmetry. In flat space the KählerDirac field can be decomposed into a set of degenerate Dirac spinors but this equivalence is lost in a curved background since the coupling to gravity differs from the Dirac case. Indeed, unlike the case of Dirac fermions, Kähler-Dirac fermions can be defined on an arbitrary manifold without the need to introduce a frame and spin connection. These facts were emphasized many years ago by Banks et al. where an attempt was made to identify the four Dirac fermions residing in each four dimensional Kähler-Dirac field with the number of generations in the Standard Model [1]. With precision Large ElectronPositron Collider data ruling out a fourth generation this idea had to be abandoned. However, in this paper, we will

Published by the American Physical Society under the terms of the Creative Commons Attribution 4.0 International license. Further distribution of this work must maintain attribution to the author(s) and the published article's title, journal citation, and DOI. Funded by SCOAP ${ }^{3}$. argue that there may be another natural interpretation for this degeneracy-it is required in order to write down anomaly free, and hence consistent, theories of interacting fermions.

These constraints arise because the partition function of a massless free Kähler-Dirac field in curved space transforms by a phase under a particular global $U(1)$ symmetry with the phase being determined by the Euler character $\chi$ of the background. The appearance of $\chi$ shows that the anomaly is gravitational in origin but is distinct from the usual mixed gravitational anomaly of Weyl fermions [2]. If we compactify flat space to a sphere this anomaly breaks the $U(1)$ to $Z_{4}$ in even dimensions which is sufficient to prohibit the appearance of fermion bilinear terms in the quantum effective action. Four fermion terms are however allowed and the simplest operator of this type requires two flavors of Kähler-Dirac fermion. Since a massless Kähler-Dirac field can be decomposed into two so-called reduced Kähler-Dirac fields, each carrying half the original degrees of freedom, four reduced fields are required for the minimal four fermion interaction.

In even dimensions a reduced Kähler-Dirac field, in the flat space limit, can be decomposed into $2^{D / 2}$ Majorana spinors and we learn that consistent interacting theories in even dimensions possess $2^{D / 2+2}$ Majorana fields. These fermion numbers agree with a series of anomaly cancellation conditions associated with certain discrete symmetries in dimensions two and four-see Table I and [3,4]. 
TABLE I. Number of Weyl fermions needed for consistent interacting theories in $D=2$ and $D=4$.

\begin{tabular}{lcc}
\hline \hline $\mathrm{D}$ & Symmetry & Critical number of Majoranas \\
\hline 2 & Chiral fermion parity & 8 \\
4 & Spin- $_{4}$ & 16 \\
\hline \hline
\end{tabular}

Since cancellation of all 't Hooft anomalies is a necessary condition for fermions to acquire mass without breaking symmetries this suggests it may be possible to build models with precisely this fermion content where all fermions are gapped in the IR. In fact there are examples in condensed matter physics where precisely this occurs, see [5-12]. Similar results have been obtained in staggered fermions in lattice gauge theory [13-18].

The plan of the paper is as follows. We start in Sec. II by giving a brief introduction to Kähler-Dirac fermions exhibiting their connection to Dirac fermions, and showing how, in the case of massless fields, the theory is invariant under a $U(1)$ symmetry whose generator $\Gamma$ anticommutes with the Kähler-Dirac operator on any curved background. Using $\Gamma$ one can project out half the degrees of freedom to obtain a reduced Kähler-Dirac field. Section III shows that the $U(1)$ symmetry suffers from a gravitational anomaly in even dimensions and breaks to $Z_{4}$. In Sec. IV we show using a spectral flow argument that this remaining $Z_{4}$ symmetry suffers from a global anomaly in the presence of interactions unless the theory contains multiples of four reduced Kähler-Dirac fields. In Sec. V we point out that a necessary condition for symmetric mass generation in such theories is that these anomalies cancel and we give examples of possible four fermion interactions that might be capable of achieving this. Section VI points out the connections between these continuum ideas and lattice fermions and a final summary appears in Sec. VII.

\section{REVIEW OF KÄHLER-DIRAC FIELDS}

The Kähler-Dirac equation arises on taking the square root of the Laplacian operator written in terms of exterior derivatives. We start by defining the Kähler-Dirac operator $K=d-d^{\dagger}$ with $d^{\dagger}$ the adjoint of $d$. Clearly $-K^{2}=d d^{\dagger}+$ $d^{\dagger} d=\square$ since the derivative operators are nilpotent. This suggests an alternative to the usual Dirac equation [19]:

$$
(K-m) \Phi=0
$$

where $\Phi=\left(\phi_{0}, \phi_{1}, \ldots \phi_{p}, \phi_{D}\right)$ is a collection of $p$ forms. The action of the derivative operators on these forms is then given by $[1,18]$

$d \Phi=\left(0, \partial_{\mu} \phi, \partial_{\mu_{1}} \phi_{\mu_{2}}-\partial_{\mu_{2}} \phi_{\mu_{1}}, \ldots, \sum_{\text {perms } \pi}(-1)^{\pi} \partial_{\mu_{1}} \phi_{\mu_{2} \ldots \mu_{D}}\right)$

$$
-d^{\dagger} \Phi=\left(\phi^{\nu}, \phi_{\mu}^{\nu}, \ldots, \phi_{\mu_{1}, \ldots, \mu_{D-1}}^{\nu}, 0\right)_{; \nu} .
$$

An inner product of two such Kähler-Dirac fields $A$ and $B$ can be defined as

$$
[A, B]=\sum_{p} \frac{1}{p !} a^{\mu_{1} \ldots \mu_{D}} b_{\mu_{1} \ldots \mu_{D}}
$$

Using this allows one to obtain the Kähler-Dirac equation by the variation of the Kähler-Dirac action

$$
S_{\mathrm{KD}}=\int d^{D} x \sqrt{g}[\bar{\Phi},(K-m) \Phi]
$$

where $\bar{\Phi}$ is an independent (in Euclidean space) KählerDirac field.

It is easy to see that the Kähler-Dirac operator anticommutes with a linear operator $\Gamma$ which acts on the $p$ form fields as

$$
\Gamma: \phi_{p} \rightarrow(-1)^{p} \phi_{p}
$$

This anticommutation property can be used to construct a $U(1)$ symmetry of the massless Kähler-Dirac action which acts on $\Phi$ as

$$
\Phi \rightarrow e^{i \alpha \Gamma} \Phi
$$

Furthermore, using $\Gamma$ one can define operators which project out even and odd form fermions-so-called reduced Kähler-Dirac fields $\Phi_{ \pm}=P_{ \pm} \Phi$ with

$$
P_{ \pm}=\frac{1}{2}(1 \pm \Gamma)
$$

The Kähler-Dirac operator $K$ couples even to odd forms and hence the massless Kähler-Dirac action separates into two independent pieces $S=\int\left(\bar{\Phi}_{+} K \Phi_{-}+\bar{\Phi}_{-} K \Phi_{+}\right)$. Retaining just one of these terms one obtains an action for such a reduced Kähler-Dirac (RKD) field

$$
S_{\mathrm{RKD}}=\int d^{D} x \sqrt{g}\left[\bar{\Phi}_{-}, K \Phi_{+}\right] .
$$

Notice that the single flavor reduced theory admits no mass term since $\bar{\Phi}_{+} \Phi_{-}=0$. Finally if we relabel $\bar{\Phi}_{-} \rightarrow \Phi_{-}$this reduced action can be rewritten in a Majorana-like form

$$
S_{\mathrm{RKD}}=\frac{1}{2} \int d^{D} x \sqrt{g}[\Phi, K \Phi] .
$$

Given that both the Dirac operator and the Kähler-Dirac operator correspond to square roots of the Laplacian one might imagine that there is a relation between the KählerDirac field and spinor fields. To exhibit this relationship we 
construct a matrix $\Psi$ by combining the $p$ form components of the Kähler-Dirac field $\Phi$ with products of Dirac gamma matrices

$$
\Psi=\sum_{p=0}^{D} \frac{1}{p !} \gamma^{\mu_{1} \ldots \mu_{p}} \boldsymbol{\phi}_{\mu_{1} \ldots \mu_{p}},
$$

where $\gamma^{\mu_{1} \ldots \mu_{p}}=\gamma^{\mu_{1}} \gamma^{\mu_{2}} \cdots \gamma^{\mu_{p}}$ are constructed using the usual (Euclidean) Dirac matrices $\gamma^{\mu}=\gamma^{a} e_{a}^{\mu}$. ${ }^{1}$ In flat space it is straightforward to show that the matrix $\Psi$ satisfies the usual Dirac equation

$$
\left(\gamma^{\mu} \partial_{\mu}-m\right) \Psi=0
$$

and describes $2^{D / 2}$ degenerate Dirac spinors corresponding to the columns of $\Psi$. This equation of motion can be derived from the action

$$
S=\int d^{D} x \operatorname{Tr}\left[\bar{\Psi}\left(\gamma^{\mu} \partial_{\mu}-m\right) \Psi\right]
$$

This action is invariant under a global $\operatorname{Spin}(D) \times S U\left(2^{D / 2}\right)$ symmetry where the first factor corresponds to Euclidean Lorentz transformations and the second to an internal flavor symmetry. In even dimensions we can write a matrix representation of the $U(1)$ generator as $\Gamma \equiv \gamma_{5} \otimes \gamma_{5}$ where the two factors act by left and right multiplication on $\Psi$. The matrix representation of a reduced Kähler-Dirac field is then given by

$$
\Psi_{ \pm}=\frac{1}{2}\left(\Psi \pm \gamma_{5} \Psi \gamma_{5}\right)
$$

Similarly the reduced action can be written as

$$
\int d^{D} x \operatorname{Tr}\left(\bar{\Psi}_{-} \gamma^{\mu} \partial_{\mu} \Psi_{+}\right)
$$

The condition $\bar{\Phi}_{-}=\Phi_{-}$then implies the matrix condition

$$
\Psi^{*}=B \Psi B^{T}
$$

where $B=C \gamma_{5}$ with $C$ the usual charge conjugation matrix. Using this one can write a Majorana-like matrix representation of the reduced action as

$$
\frac{1}{2} \int d^{D} x \operatorname{Tr}\left(B \Psi^{T} B^{T} \gamma^{\mu} \partial_{\mu} \Psi\right)
$$

\footnotetext{
${ }^{1}$ We will restrict ourselves to even dimensions in what follows. Odd dimensions require twice as many spinor degrees of freedom to match the number of components of a Kähler-Dirac field-see [20].
}

Notice that after this reduction the free theory in flat space corresponds to $2^{D / 2-1}$ Dirac or $2^{D / 2}$ Majorana spinors.

We can gain further insight into the reality condition Eq. (16) by going to a chiral basis for the gamma matrices. In four dimensions the full Kähler-Dirac field $\Psi$ then takes the form

$$
\Psi=\left(\begin{array}{cc}
E & O^{\prime} \\
O & E^{\prime}
\end{array}\right)
$$

where $O$ and $O^{\prime}$ denote $2 \times 2$ blocks of odd form fields while $E$ and $E^{\prime}$ denote corresponding even form fields. Each block contains a doublet of Weyl fields which transform in representations of the $S U(2)_{L} \times S U(2)_{R}$ Lorentz and an $S U(2) \times S U(2)$ flavor symmetry. The condition $\Psi^{*}=$ $B \Psi B^{T}$ implies $O^{\prime}=i \sigma_{2} O^{*} i \sigma_{2}$ and $E^{\prime}=-i \sigma_{2} E^{*} i \sigma_{2}$. This suggests that the operation $X \rightarrow i \sigma_{2} X^{*} i \sigma_{2}$ can be interpreted as a generalized charge conjugation operator that flips both chirality and flavor representation of a given Weyl doublet within the Kähler-Dirac field. It also implies that both $\left(O, O^{\prime}\right)$ and $\left(E, E^{\prime}\right)$ constitute doublets of Majorana spinors.

Finally we should note that while the Kähler-Dirac equation Eq. (1) written in the language of forms does not change in curved space, its matrix representation takes the modified form

$$
\left(e_{a}^{\mu} \gamma^{a} D_{\mu}-m\right) \Psi=0
$$

where $e_{\mu}^{a}$ is the vielbein or frame and $D_{\mu} \Psi=\partial_{\mu} \Psi+$ $\left[\omega_{\mu}, \Psi\right]$ is the covariant derivative associated to the spin connection.

\section{A GRAVITATIONAL ANOMALY FOR KÄHLER-DIRAC FIELDS}

In the previous section we showed that the Kähler-Dirac action is invariant under a $U(1)$ symmetry. However in the quantum theory we should also be careful to examine the invariance of the fermion measure. We will do this for a generic four dimensional curved background using the matrix representation of the Kähler-Dirac theory. The curved space action in $D=4$ reads

$$
S=\int d^{4} x \sqrt{g} \operatorname{Tr}\left(\bar{\Psi} e_{a}^{\mu} \gamma^{a} D_{\mu} \Psi\right)
$$

where $D_{\mu} \Psi=\partial_{\mu} \Psi+\left[\omega_{\mu}, \Psi\right]$ with $\omega_{\mu}$ the spin connection and we have introduced the frame field $e_{\mu}^{a}(x)$ to translate between flat and curved space indices with $e_{\mu}^{a} e_{\nu}^{b} \delta_{a b}=g_{\mu \nu}$. In the standard way we start by expanding $\Psi$ and $\bar{\Psi}$ on a basis of eigenstates of the Kähler-Dirac operator:

$$
\gamma^{\mu} D_{\mu} \phi_{n}=\lambda_{n} \phi_{n}
$$


with $\int d^{4} x e(x) \operatorname{Tr}\left(\bar{\phi}_{n}(x) \phi_{m}(x)\right)=\delta_{n m}$ and $e=\operatorname{det}\left(e_{\mu}^{a}\right)$. Thus

$$
\begin{aligned}
& \Psi(x)=\sum_{n} a_{n} \phi_{n}(x), \\
& \bar{\Psi}(x)=\sum_{n} \bar{b}_{n} \bar{\phi}_{n}(x) .
\end{aligned}
$$

The measure is then written $D \bar{\Psi} D \Psi=\prod_{n} d \bar{b}_{n} d a_{n}$ and the variation of this measure under the $U(1)$ transformation with parameter $\alpha(x)$ is given by $e^{-2 i \int \alpha(x) A(x)}$ where the anomaly $A$ is formally given by

$$
A(x)=\operatorname{Tr} \sum_{n} e \bar{\phi}_{n} \Gamma \phi_{n}(x)
$$

where the operator $\Gamma=\Gamma_{5} \otimes \gamma_{5}$ carries a flavor rotation matrix $\gamma_{5}$ acting on the right of the matrix field and a curved space chiral matrix $\Gamma_{5}$ acting on the left with $\Gamma^{5}=\gamma^{a} \gamma^{b} \gamma^{c} \gamma^{d} e_{a}^{1} e_{b}^{2} e_{c}^{3} e_{d}^{4}=\gamma^{5} e$. We need a gauge invariant regulator for this expression so we try inserting the factor

$$
e^{\frac{1}{M^{2}}\left(\gamma^{\mu} D_{\mu}\right)^{2}}
$$

into the expression for $A$. We can write

$$
\left(\gamma^{\mu} D_{\mu}\right)^{2}=D^{\mu} D_{\mu}+e_{c}^{\mu} e_{d}^{\nu} \sigma^{c d}\left[D_{\mu}, D_{\nu}\right]
$$

where $\sigma^{c d}=\frac{1}{4}\left[\gamma^{c}, \gamma^{d}\right]$ are the generators of $\operatorname{spin}(4)$. Furthermore for KD fermions we have:

$$
\left[D_{\mu}, D_{\nu}\right] \psi=\left[R_{\mu \nu}, \psi\right]
$$

where $R_{\mu \nu}=\frac{1}{2} R_{\mu \nu}^{a b} \sigma_{a b}$. Plugging this expression into Eq. (26) yields

$$
\left(\gamma^{\mu} D_{\mu}\right)^{2} \psi=D^{\mu} D_{\mu} \psi+\frac{1}{2} e_{c}^{\mu} e_{d}^{\nu} \sigma^{c d} R_{\mu \nu}^{a b}\left[\sigma_{a b}, \psi\right] .
$$

The anomaly can then be written

$$
\begin{aligned}
A(x)= & \lim _{M \rightarrow \infty} \operatorname{Tr} \sum_{n} e\left(\bar{\phi}_{n} \Gamma e^{\frac{1}{M^{2}}\left(\gamma^{\mu} D_{\mu}\right)^{2}} \phi_{n}\right) \\
= & \lim _{M \rightarrow \infty} \operatorname{Tr}\left(\Gamma e^{\frac{1}{M^{2}}\left(\gamma^{\mu} D_{\mu}\right)^{2}} \sum_{n} e \phi_{n} \bar{\phi}_{n}\right) \\
= & \lim _{x \rightarrow x^{\prime} M \rightarrow \infty} \lim _{M \rightarrow} \operatorname{Tr}\left(\Gamma e^{\frac{1}{M^{2}}\left(\gamma^{\mu} D_{\mu}\right)^{2}} \delta\left(x-x^{\prime}\right)\right) \\
= & \lim _{x \rightarrow x^{\prime} M \rightarrow \infty} \lim _{i} \operatorname{Tr}\left(e \gamma^{5} e^{\frac{1}{M^{2}}\left(D^{\mu} D_{\mu}+\frac{1}{2} e_{c}^{\mu} e_{d}^{l} c^{c d} R_{\mu \nu}^{a b}\left[\sigma_{a b}, \cdot\right]\right)}\right. \\
& \left.\times \delta\left(x-x^{\prime}\right) \gamma_{5}\right) .
\end{aligned}
$$

Expanding the exponential to $O\left(1 / M^{4}\right)$ to get a nonzero result for the trace over spinor and flavor indices and acting with $e^{\frac{1}{M^{2}} D_{\mu} D^{\mu}}$ on the delta function yields ${ }^{2}$

$$
\begin{aligned}
A= & \frac{1}{16 \pi^{2}}\left(\frac{1}{2 !}\right)\left(\frac{1}{4}\right) \operatorname{tr}\left(e \gamma^{5} \sigma^{a b} \sigma^{c d}\right) e_{a}^{\mu} e_{b}^{\nu} e_{c}^{\rho} e_{d}^{\lambda} R_{\mu \nu}^{C D} R_{\rho \lambda}^{E F} \\
& \times \operatorname{tr}\left(\sigma_{C D} \sigma_{E F} \gamma_{5}\right) \\
= & \frac{1}{128 \pi^{2}} \epsilon^{\mu \nu \rho \lambda} \epsilon_{C D E F} R_{\mu \nu}^{C D} R_{\rho \lambda}^{E F}
\end{aligned}
$$

where we have also employed the result:

$$
e \epsilon^{a b c d} e_{a}^{\mu} e_{b}^{\nu} e_{c}^{\rho} e_{d}^{\lambda}=\epsilon^{\mu \nu \rho \lambda} .
$$

Thus the anomaly $A(x)$ is just the Euler density and we find that the phase transformation of the partition function under the global $U(1)$ symmetry is then

$$
Z \rightarrow e^{-2 i \alpha \int d^{4} x A(x)}=e^{-2 i \alpha \chi} Z .
$$

This result for the anomaly agrees with a previous lattice calculation that employed a discretization of the KählerDirac action on simplicial lattices [18]. The nonzero value for the anomaly originates in the existence of exact zero modes of the Kähler-Dirac operator. Such zero modes are eigenstates of $\Gamma$ and Eq. (24) shows that $\int d^{4} x A(x)=$ $n_{+}-n_{-}$where $n_{ \pm}$denotes the number of zero modes with $\Gamma= \pm 1$. Our final result is then a consequence of the index theorem

$$
n_{+}-n_{-}=\chi
$$

On the sphere (which can be regarded as a compactification of $R^{4}$ ) the presence of this phase breaks the $U(1)$ to $Z_{4}$. This nonanomalous $Z_{4}$ is then sufficient to prohibit fermion bilinear mass terms from appearing in the effective action of the theory. This compactification of the space on a sphere is similar to the strategy that is used to show the importance of instantons in the QCD path integral. It places constraints on the terms that can appear in the effective action as the radius of the sphere is sent to infinity. Four fermion terms are allowed but require at least two flavors of Kähler-Dirac field to be nonvanishing. ${ }^{3}$ Since each massless Kähler-Dirac field can be written in terms of two independent reduced fields this implies consistent, interacting theories require four reduced Kähler-Dirac fields. In four dimensions, and taking the flat space limit,

\footnotetext{
${ }^{2}$ See Appendix for more details.

${ }^{3}$ Of course we can go further and demand that the anomaly be canceled for manifolds with other values of $\chi$. For example, on manifolds with $\chi=-4$ only eight fermion terms are allowed, only twelve fermion terms on spaces with $\chi=-6$ etc. Other work on higher order multifermion interactions can be found in [21,22].
} 
each such reduced field corresponds to four Majorana fermions and we learn that such theories contain sixteen Majorana fermions. It is not hard to generalize this argument to any even dimension.

\section{A GLOBAL $Z_{4}$ ANOMALY}

In the last section we found that a system of free KählerDirac fermions propagating on an even dimensional space is anomalous and remains invariant only under a $Z_{4}$ symmetry. In this section we will examine such a theory in the presence of interactions and show that this residual $Z_{4}$ symmetry suffers from a global anomaly unless the theory contains multiples of four reduced Kähler-Dirac fields.

From Eq. (10) it is clear that the effective action for a reduced Kähler-Dirac fermion is given by a $\operatorname{Pfaffian} \operatorname{Pf}(K)$. From the property $[\Gamma, K]_{+}=0$ it is easy to show that $\operatorname{det}(K) \geq 0$. However since $\operatorname{Pf}(K)= \pm \sqrt{\operatorname{det}}(K)$ there is an ambiguity in the phase of the Pfaffian.

To analyze this in more detail we will consider the theory on the nonorientable manifold $R P^{4}$ and deform the theory to remove the one zero mode. The simplest possibility is to couple a pair of such reduced Kähler-Dirac fields to an auxiliary real scalar field $\sigma$. The fermion operator is then given by

$$
M=\delta^{a b} K+\sigma(x) \epsilon^{a b} .
$$

We will assume that the total action (including terms involving just $\sigma$ ) is invariant under a discrete symmetry which extends the fermionic $Z_{4}$ discussed in the previous section:

$$
\begin{gathered}
\Phi \rightarrow i \Gamma \Phi, \\
\sigma \rightarrow-\sigma .
\end{gathered}
$$

Notice that this fermion operator is antisymmetric and real and hence all eigenvalues of $M$ lie on the imaginary axis. Let us define the Pfaffian as the product of the eigenvalues in the upper half plane in the background of some reference configuration $\sigma=\sigma_{0}=$ constant. By continuity we define the Pfaffian to be the product of these same eigenvalues under fluctuations of $\sigma$. Furthermore, it is easy to see that as a consequence of the $Z_{4}$ symmetry

$$
\Gamma M(\sigma) \Gamma=-M(-\sigma) .
$$

This result shows that the spectrum and hence the determinant is indeed invariant under the $Z_{4}$ transformation $\sigma \rightarrow-\sigma$. But this is not enough to show the Pfaffian itself is unchanged since there remains the possibility that eigenvalues flow through the origin as $\sigma$ is deformed smoothly to $-\sigma$ leading to a sign change. To understand what happens we consider a smooth interpolation of $\sigma$ :

$$
\sigma(s)=s \sigma_{0} s \in(-1,+1) .
$$

The question of eigenvalue flow can be decided by focusing on the behavior of the eigenvalues of the fermion operator closest to the origin at small $s$. In this region the eigenvalues of smallest magnitude correspond to fields which are constant over the lattice and satisfy the eigenvalue equation:

$$
\sigma_{0} s \epsilon^{a b} v^{b}=\lambda v^{a} .
$$

The two eigenvalues $\lambda= \pm i \sigma_{0} s$. Clearly these eigenvalues change sign as $s$ varies from positive to negative values leading to a Pfaffian sign change. This can also be seen explicitly from Eq. (34) since

$$
\operatorname{Pf}[M(-\sigma)]=\operatorname{det}[\Gamma] \operatorname{Pf}[M(\sigma)]=-\operatorname{Pf}[M(\sigma)] .
$$

We thus learn that the Pfaffian of the two flavor system indeed changes sign under the $Z_{4}$ transformation. On integration over $\sigma$ the value of any even function of $\sigma$ including the partition function would then yield zero rendering expectation values of $Z_{4}$ invariant operators ill defined. This corresponds to a mixed global 't Hooft anomaly between the discrete $Z_{4}$ symmetry and a gauged $Z_{2}$ reflection symmetry in the interacting theory.

Clearly this global anomaly will be canceled for any multiple of four reduced Kähler-Dirac fields provided that they couple via a Yukawa term of the form $\sigma(x) \Phi^{a} C^{a b} \Phi^{b}$ with $C$ a real, antisymmetric matrix. In that case $C$ can be brought to a canonical antisymmetric form $\left(\lambda_{1} i \sigma_{2} \otimes \lambda_{2} i \sigma_{2} \otimes \ldots\right)$ using a nonanomalous orthogonal transformation. Positivity of the Pfaffian under $\sigma \rightarrow-\sigma$ then depends on the Yukawa interaction in $M$ containing an even number of such $2 \times 2$ blocks. Decomposing the reduced Kähler-Dirac field in a flat background into spinors we see that anomaly cancellation occurs for eight or sixteen Majorana fermions in two and four dimensions respectively.

The spectral flow argument we have given is similar to the one given by Witten in showing that a single Weyl fermion in the fundamental representation of $S U(2)$ is anomalous [23].

\section{SYMMETRIC MASS GENERATION}

The cancellation of anomalies is crucial to the problem of giving masses to fermions without breaking symmetries. Since anomalies originate from massless states then any phase where all states are massive in the IR must necessarily arise from a UV theory with vanishing anomaly. In particular, it is only possible to accomplish such symmetric mass generation if one cancels off the 't Hooft anomalies for all global symmetries [24,25].

In the previous section we have seen that only multiples of four reduced Kähler-Dirac fields have vanishing $Z_{4}$ 
anomaly. Thus we require that any interactions we introduce in the theory respect this symmetry. The simplest such interaction is a four fermion operator as we have already discussed. It corresponds to adding a simple $\int \sigma^{2}$ term to the Yukawa action discussed in the previous section.

We should note however that cancellation of anomalies is a necessary condition for symmetric mass generation but it may not be sufficient - the fact that four fermion terms are perturbatively irrelevant operators in dimensions greater than two may mean that a more complicated scalar action is required-indeed this was the finding of numerical work in four dimensions where a continuous phase transition to a massive symmetric phase was found only by tuning an additional scalar kinetic term [26].

With this caveat it is useful to give examples of possible four fermion terms that might lead to symmetric mass generation For example, one can imagine taking four reduced Kähler-Dirac fields transforming in the fundamental representation of a $S O(4)$ symmetry and employ the term

$$
\int d^{D} x \sqrt{g}\left(\left[\Phi^{a}, \Phi^{b}\right]_{+}\right)^{2}
$$

where the + subscript indicates that fermion bilinear is projected to the self-dual $(1,0)$ representation of $S O(4)$. In practice this can be implemented in flat space via a Yukawa term which is given in the matrix representation by

$$
\int d^{D} x G \operatorname{Tr}\left(\Psi^{a}(x) \Psi^{b}(x)\right)_{+} \sigma_{a b}(x)+\frac{1}{2} \sigma_{a b}^{2}(x) .
$$

Notice that this Yukawa interaction is mediated by a scalar $\sigma_{a b}^{+}$that also transforms in the self-dual representation of $S O(4)$

$$
\sigma_{a b}^{+}=\frac{1}{2}\left(\sigma_{a b}+\frac{1}{2} \epsilon_{a b c d} \sigma_{c d} .\right)
$$

In the next section we show how Kähler-Dirac theories can be discretized in a manner which leaves the anomaly structure of the theory intact and results in theories of (reduced) staggered fermions. The four fermion interaction that results from integrating over $\sigma_{+}$in Eq. (39) has been studied using numerical simulation and the results of this work indeed provide evidence of a massive symmetric phase in dimensions two and three [13-16] while an additional scalar kinetic operator was also needed in four dimensions [26].

As another example one can take eight flavors of reduced Kähler-Dirac field which are taken to transform in the eight dimensional real spinor representation of $\operatorname{spin}(7)$. An appropriate Yukawa term which might be used to gap those fermions is given by

$$
\int d^{D} x \operatorname{Tr}\left(\Psi^{a}(x) \Gamma_{\mu}^{a b} \Psi^{b}(x)\right) \sigma_{\mu}(x)
$$

where $\Gamma_{\mu}, \mu=1 \ldots 7$ are the (real) Dirac matrices for $\operatorname{spin}(7)$ [8]. This interaction was shown by Fidkowski and Kitaev to gap out boundary Majorana modes in a $(1+1)$-dimensional system without breaking symmetries [5]. This interaction may also play a role in constructing Kähler-Dirac theories that target grand unified theory (GUT) models. For example, if one is able to gap out the $\left(E, E^{\prime}\right)$ blocks occurring in Eq. (18) for a reduced Kähler-Dirac field valued in $\operatorname{spin}(7)$ the remaining light fields live in the representation $(8,2,1)$. If the $\operatorname{spin}(7)$ is subsequently Higgsed to $\operatorname{spin}(6)=S U(4)$ then this representation breaks to $(4,2,1) \oplus(\overline{4}, 1,2)$ which is the field content of the Pati-Salam theory [27].

\section{EXACT ANOMALIES FOR LATTICE FERMIONS}

One of the most important properties of the Kähler-Dirac equation is that it can be discretized without encountering fermion doubling $[28,29]$. Furthermore this discretization procedure can be done for any random triangulation of the space allowing one to capture topological features of the spectrum. The idea is to replace continuum $p$ forms by $p$ cochains or lattice fields living on oriented $p$ simplices in the triangulation. The exterior derivative and its adjoint are mapped to coboundary and boundary operators which act naturally on these $p$-simplices and retain much of the structure of their continuum cousins-for example they are both nilpotent operators. Homology theory can then be used to show that the spectrum of the discrete theory evolves smoothly into its continuum cousin as the lattice spacing is sent to zero - there are no additional lattice modes or doublers that obscure the continuum limit. Furthermore, the number of exact zero modes of the lattice Kähler-Dirac operator is exactly the same as found in the continuum theory. This immediately suggests that the anomaly encountered earlier which depends only on the topology of the background space can be exactly reproduced in the lattice theory. This was confirmed in [18] where a lattice calculation revealed precisely the same gravitational anomaly derived in this paper.

If one restricts to regular lattices with the topology of the torus it is straightforward to see that the discrete KählerDirac operator discussed above can be mapped to a staggered lattice fermion operator on a lattice with half the lattice spacing [1]. One simply maps the p-form components located in the matrix $\Psi^{a}$ into a set of single component lattice fermions $\chi^{a}$ via

$$
\Psi(x)=\sum_{n_{\mu}=0,1} \chi\left(x+n_{\mu}\right) \gamma^{\left(x+n_{\mu}\right)}
$$

where $\gamma^{x}=\prod_{i=1}^{D} \gamma_{i}^{x_{i}}$ and the summation runs over the $2^{D}$ points in a unit hypercube of a regular lattice. If one 
substitutes this expression into the continuum kinetic term, replaces the continuum derivative with a symmetric finite difference and carries out the trace operation one obtains the free staggered fermion action. Indeed the operator $\Gamma$ acting on forms then becomes the site parity operator $\epsilon(x)=(-1)^{\sum_{i=1}^{D} x_{i}}$ and the $U(1)$ symmetry of the massless Kähler-Dirac action is just the familiar $U(1)_{\epsilon}$ symmetry of staggered fermions. Indeed, it is possible to repeat the arguments of section IV to show that a staggered fermion theory equipped with a four fermion term is only well defined for multiples of four reduced staggered fermions under which the classical $Z_{4}$ symmetry is preserved. This helps to explain why these theories seem capable of generating a massive symmetric phase [13-16,26].

\section{SUMMARY}

In this paper we have shown that theories of massless Kähler-Dirac fermions suffer from a gravitational anomaly that breaks a $U(1)$ symmetry down to $Z_{4}$ in even dimensions. We derive this anomaly by computing the symmetry variation of the path integral for free Kähler-Dirac fermions propagating in a background curved space. The remaining $Z_{4}$ prohibits fermion bilinear mass terms from arising in the quantum effective action. We then use spectral flow arguments to argue that multiples of four flavors of KählerDirac are needed to avoid a further global anomaly in this $Z_{4}$ symmetry in the presence of interactions. Since four fermion interactions are allowed by these constraints we argue that they may be capable of gapping such systems without breaking symmetries.

In flat space each reduced Kähler-Dirac field transforming in a real representation can be decomposed into $2^{D / 2}$ Majorana fermions. Thus anomaly cancellation in the interacting theory dictates a very specific fermion content-multiples of eight and sixteen Majorana fermions in two and four dimensions respectively. Remarkably, this fermion counting agrees with independent constraints based on the cancellation of the chiral fermion parity and spin- $Z_{4}$ symmetries of Weyl fermions in two and four dimensions $[3,11]$. $^{4}$

Finally we discuss how this anomaly can be realized exactly in lattice realizations of such systems and emphasize how the results in this paper shed light on the appearance of massive symmetric phases in recent simulations of lattice four fermion models. The appearance of an anomaly in a lattice system is notable as it contradicts the usual folklore that anomalies only appear in systems with an infinite number of degrees of freedom.

\footnotetext{
${ }^{4}$ One can also decompose a Kähler-Dirac fermion into two and four Majorana spinors in one and three dimensions respectively. Building four fermion operators for these Kähler-Dirac fields then yields theories with eight and sixteen Majorana spinors which is also in agreement with results from odd dimensional topological insulators.
}

While the anomaly vanishes for closed odd dimensional manifolds it is nonzero for odd dimensional manifolds with boundary. For example the Euler characteristic of the three ball is $\chi\left(B^{3}\right)=1$ and the symmetry in the bulk is hence broken to $Z_{2}$ allowing for the presence of mass terms. However the boundary fields living on $S^{2}$ possess an enhanced $Z_{4}$ symmetry prohibiting such bilinear terms and we learn that such boundary fields can instead be gapped using four fermion interactions.

\section{ACKNOWLEDGMENTS}

This work was supported by the U.S. Department of Energy (DOE), Office of Science, Office of High Energy Physics under Award No. DE-SC0009998. S. C. is grateful for helpful discussions with Erich Poppitz, David Tong and Yuven Wang.

\section{APPENDIX: DELTA FUNCTION}

Following [2]

$$
\delta\left(x-x^{\prime}\right)=\int \frac{d^{4} k}{(2 \pi)^{4}} e^{i k_{\mu} D^{\mu} \sigma\left(x, x^{\prime}\right)}
$$

where $D_{\mu}$ is now the Kähler-Dirac operator and $\sigma\left(x, x^{\prime}\right)$ is the geodesic biscalar [a generalization of $\frac{1}{2}\left(x-x^{\prime}\right)^{2}$ in flat space] defined by

$$
\sigma\left(x, x^{\prime}\right)=\frac{1}{2} g^{\mu \nu} D_{\mu} \sigma\left(x, x^{\prime}\right) D_{\nu} \sigma\left(x, x^{\prime}\right)
$$

with

$$
\sigma(x, x)=0
$$

and

$$
\lim _{x \rightarrow x^{\prime}} D_{\mu} D^{\nu} \sigma\left(x, x^{\prime}\right)=g_{\mu}^{\nu} .
$$

Now,

$$
\begin{aligned}
D^{2} \delta\left(x-x^{\prime}\right)= & D^{\nu} \int \frac{d^{4} k}{(2 \pi)^{4}}\left[i k_{\lambda} D_{\nu} D^{\lambda} \sigma\right. \\
& +\frac{1}{2 !}\left\{\left(D_{\nu}(i k \cdot D \sigma)\right)(i k \cdot D \sigma)\right. \\
& \left.+(i k \cdot D \sigma)\left(D_{\nu}(i k \cdot D \sigma)\right\}+\cdots\right] \\
= & \int \frac{d^{4} k}{(2 \pi)^{4}}\left[D^{\nu}\left(i k_{\lambda} D_{\nu} D^{\lambda} \sigma\right)\right. \\
& \left.+\left(i k_{\lambda} D_{\nu} D^{\lambda} \sigma\right)\left(i k_{\rho} D^{\nu} D^{\rho} \sigma\right)+\cdots\right] .
\end{aligned}
$$


Taking $\lim _{x \rightarrow x^{\prime}}$, other terms represented by ... vanish, and we obtain

$$
\begin{aligned}
D^{2} \delta\left(x-x^{\prime}\right) & =\int \frac{d^{4} k}{(2 \pi)^{4}}\left[\left(i k_{\lambda} g_{\nu}^{\lambda}\right)\left(i k_{\rho} g^{\nu \rho}\right)+D^{\nu}\left(i k_{\lambda} g_{\nu}^{\lambda}\right)\right] \\
& =\int \frac{d^{4} k}{(2 \pi)^{4}}\left[\left(i k_{\nu}\right)\left(i k^{\nu}\right)+i D^{\nu}\left(k_{\nu}\right)\right] \\
& =\int \frac{d^{4} k}{(2 \pi)^{4}}\left[-k^{2}+i D^{\nu}\left(k_{\nu}\right)\right]
\end{aligned}
$$

Which implies

$$
\lim _{x \rightarrow x^{\prime}} e^{D^{2}} \delta\left(x-x^{\prime}\right)=\int \frac{d^{4} k}{(2 \pi)^{4}} e^{\left[-k^{2}+i D^{\nu}\left(k_{\nu}\right)\right]} .
$$

Hence,

$$
\begin{aligned}
\lim _{x \rightarrow x^{\prime}} e^{D^{2} / M^{2}} \delta\left(x-x^{\prime}\right) & =\int \frac{d^{4} k}{(2 \pi)^{4}} e^{\left[-k^{2}+i D^{\nu}\left(k_{\nu}\right)\right] / M^{2}} \\
& =M^{4} \int \frac{d^{4} k}{(2 \pi)^{4}} e^{\left[-k^{2}+i D^{\nu}\left(k_{\nu}\right) / M\right]} \\
& =M^{4} \frac{1}{16 \pi^{2}} .
\end{aligned}
$$

[1] T. Banks, Y. Dothan, and D. Horn, Phys. Lett. 117B, 413 (1982).

[2] K. Fujikawa, Phys. Rev. D 21, 2848 (1980).

[3] I. n. García-Etxebarria and M. Montero, J. High Energy Phys. 08 (2019) 003.

[4] Z. Wan and J. Wang, J. High Energy Phys. 07 (2020) 062.

[5] L. Fidkowski and A. Kitaev, Phys. Rev. B 81, 134509 (2010).

[6] S. Ryu and S.-C. Zhang, Phys. Rev. B 85, 245132 (2012).

[7] A. Kapustin, R. Thorngren, A. Turzillo, and Z. Wang, J. High Energy Phys. 12 (2015) 052.

[8] Y.-Z. You and C. Xu, Phys. Rev. B 91, 125147 (2015).

[9] T. Morimoto, A. Furusaki, and C. Mudry, Phys. Rev. B 92, 125104 (2015).

[10] Y.-Z. You, Y.-C. He, C. Xu, and A. Vishwanath, Phys. Rev. X 8, 011026 (2018).

[11] J. Wang and X.-G. Wen, Phys. Rev. Research 2, 023356 (2020).

[12] M. Guo, K. Ohmori, P. Putrov, Z. Wan, and J. Wang, Commun. Math. Phys. 376, 1073 (2020).

[13] V. Ayyar and S. Chandrasekharan, Phys. Rev. D 91, 065035 (2015).

[14] V. Ayyar and S. Chandrasekharan, Phys. Rev. D 93, 081701 (2016).

[15] V. Ayyar and S. Chandrasekharan, J. High Energy Phys. 10 (2016) 058.
[16] S. Catterall, J. High Energy Phys. 01 (2016) 121.

[17] S. Catterall and N. Butt, Phys. Rev. D 97, 094502 (2018).

[18] S. Catterall, J. Laiho, and J. Unmuth-Yockey, J. High Energy Phys. 10 (2018) 013.

[19] E. Kahler, Rend. Math 3-4 21, 425 (1962).

[20] S. Hands, Symmetry 13, 1523 (2021).

[21] X.-C. Wu, Y. Xu, C.-M. Jian, and C. Xu, Phys. Rev. B 100, 155138 (2019).

[22] C.-M. Jian and C. Xu, Phys. Rev. B 101, 035118 (2020).

[23] E. Witten, Phys. Lett. 117B, 324 (1982).

[24] S. S. Razamat and D. Tong, Phys. Rev. X 11, 011063 (2021).

[25] D. Tong, arXiv:2104.03997.

[26] N. Butt, S. Catterall, and D. Schaich, Phys. Rev. D 98, 114514 (2018).

[27] A. Pati and J. C. Salam, Phys. Rev. D 10, 275 (1974).

[28] J. M. Rabin, Nucl. Phys. B201, 315 (1982).

[29] P. Becher and H. Joos, Z. Phys. C 15, 343 (1982).

Correction: Minor modifications in wording have been made in the last sentence of the first paragraph of Sec. IV, the first sentence of the paragraph containing Eq. (31), and the last sentence of the paragraph containing Eq. (37). 